\title{
Plasmon resonances of graphene-dielectric-metal structures calculated by the method of recurrence relations
}

Gerardo Gonzalez de la Cruz ( $\sim$ bato@fis.cinvestav.mx )

CINVESTAV IPN: Centro de Investigacion y de Estudios Avanzados del Instituto Politecnico Nacional https://orcid.org/0000-0001-6710-3424

Maurice Oliva-Leyva

CINVESTAV IPN: Centro de Investigacion y de Estudios Avanzados del Instituto Politecnico Nacional

\section{Research Article}

Keywords: recurrence relations, plasmons, metamaterials, sensors

Posted Date: February 12th, 2021

DOI: https://doi.org/10.21203/rs.3.rs-158100/v1

License: (c) (i) This work is licensed under a Creative Commons Attribution 4.0 International License.

Read Full License 


\title{
Plasmon resonances of graphene-dielectric-metal structures calculated by the method of recurrence relations
}

\author{
Gerardo Gonzalez de la Cruz* and Maurice Oliva-Leyva \\ Departamento de Fisica, CINVESTAV-IPN Apartado Postal 14-740, 07000 Ciudad de \\ Mexico, Mexico
}

\begin{abstract}
Graphene supports surface plasmons in the therahertz range, and compared with noblemetal plasmons, they show an extreme level of field confinement and relatively long propagation distances, with the advantage of being highly tunable via electrostatic field. Nevertheless, its interaction with light is normally rather weak. To obtain a more powerful capability of excite plasmons, a combination of graphene and artificial structures (metamaterials) present a powerful tunability for enhancing light-matter interaction. These features make graphene metamaterials a promising candidate for plasmonics and surface plasmon resonance for biological sensors. In this work, we study the plasmon spectra in a finite number of graphene layers on a metallic-dielectric substrate surrounded by materials with different dielectric constants. It is shown that using standard electromagnetic boundary conditions and solving the recurrence relation (a suitable alternative to transfer matrix method) for the coefficients of the electric potential between graphene layers, an explicit effective dielectric function of the metamaterial can be obtained giving the plasmon dispersion relations. It is found that the metal-dielectriclayered graphene structure supports both, high-energy optical plasmons oscillations and out-of-phase low energy acoustic charge density excitations. Experimentally, the Kretschmann configuration can be used to excite the surface plasmon resonances. It is based on the observation of a sharp minimum in the reflection coefficient versus angle (or wavelength) curve.
\end{abstract}

Keywords: recurrence relations, plasmons, metamaterials, sensors

${ }^{*}$ Correspondence: bato@fis.cinvestav.mx 


\section{Introduction}

Surface waves propagating along the boundary between a metal and dielectric medium are called surface plasmons. Since surface plasmon induce fluctuations of charge at the surface, they are accompanied by electromagnetic oscillations and exhibit confinement of the electric field in the direction normal to a conductor-dielectric interface. Furthermore, the resonant interaction between surface plasmons and the electromagnetic radiation at dielectric-metal interface results in a remarkable enhanced optical field making plasmons extremely sensitive to dielectric permittivity of the surrounding medium. The ability of controlling strong light-matter interaction through surface plasmons in metals has driven the field of plasmonics. Plasmonics is a rapidly growing discipline in fundamental research and promising applications in a variety of surface-plasmon-based-devices for optics in integrated photonic systems, solar cells, plasmonics in biology and medicine, sensors, thermo plasmonics and nonlinear phenomena [1-5]. Additionally, increasing research has been carried out to investigate how excite and manipulate surface plasmon efficiently. One possible solution for this problem consists in involving metamaterials, or artificial materials composed of suitably arranged metal-dielectric stacks, which can provide control of surface plasmons for new sensing functionalities [6]. However, the field confinement of these reported metamaterials are relatively weak and exhibit high intrinsic dissipative losses with strong damping of surface plasmons and slow damage by chemical action [7]. To overcome these shortcomings, improvement research of new physical mechanisms and better electronic and optical properties has been achieve for new plasmonic materials, among them, graphene has emerged as an alternative, unique twodimensional material able to extend the field of plasmonics for terahertz to mid-infrared applications [8-11]. Graphene, a two-dimensional material made of carbon atoms arranged in hexagonal lattice has attracted tremendous attention due to its physical properties including high electrical and thermal conductivity, optical transparency and controllable plasmon properties. Either chemical doping or electrostatic gating can modulate the carrier density in graphene and, thus the Fermi energy. Therefore, these 
excellent properties make graphene a favorable candidate for novel plasmonics devices and potential applications in photonics, optoelectronics and in sensor technologies [13]. In monolayer graphene layer, its interaction with light needs to be further enhanced to achieve adequate efficiency for practical device applications. Toward this goal, artificial engineering structures, or metamaterials, have been employed as a platform for boosting light-matter interaction in the last years $[14,25]$. Therefore, the hybridization of graphene-metal metamaterials plays an important role in the area of plasmonics, and exploring the interactions of the plasmon modes in multilayer graphene structures coupled via Coulomb interaction with metallic substrates offer new opportunities for applications and fundamental studies of collective electron excitations in plasmonic metamaterials for sensing purposes [26-29].

In this paper, we report a theoretical investigation of surface plasmon excitation in a metamaterial consisting of $\mathrm{N}$-graphene layers embedded in a medium of dielectric constant $\varepsilon_{0}$ on metallic film spatially separated by a dielectric spacer and surrounded by semi-infinite materials of dielectric constants $\varepsilon_{1}$ and $\varepsilon_{2}$, respectively, see Fig.1. Solving Poisson equation for the electric potential and applying standard boundary conditions at the interfaces between the different layers, the amplitudes associated with the electric potential between different layers satisfy a linear recurrence relation of second order and consequently, an analytical expression for the dielectric function of the metamaterial can be obtained. The zeros of the dynamic dielectric function lead the dispersion relation of the plasmon modes i.e., $\boldsymbol{\varepsilon}(\boldsymbol{q}, \boldsymbol{\omega})=\mathbf{0}$, see Eq. (10) in the text. It is possible to obtain a mathematical expression for the surface plasmon resonance using the long wavelength approximation in the dielectric function i.e., $q->0$, where $\boldsymbol{q}$ is the wave vector associated to propagation of plasmon oscillations on surface graphene. It is important to mention that in the past years the transfer matrix method has become a standard theoretical model to study optical properties and charge density excitations in non-interacting graphene layers. Thus, the recurrence method offers an alternative method to study plasmon modes in multilayer structures

\section{Theoretical approach}


In our formulation, we consider a periodic system consisting of a finite graphene monolayer stack; each layer with an electron density $n$ spatially separated a distance $d$ on a substrate constituted by a dielectric spacing layer and an optically thick metal film. The layered graphene system is represented by an array of $N$ graphene layers located ay $z=l d$ $(I=0,1, \ldots, N-1)$ inserted in a material with background dielectric constant $\varepsilon_{0}$. The substrate constituted by a metal-dielectric composite films occupy the space $-\left(d_{m}+d_{s}\right)<z<-d_{s}$ and $d_{s}<z<0$, respectively. In Fig.1 we show the graphene-dielectric-metal metamaterial surrounded by a semi-infinite materials with dielectric constants $\varepsilon_{1}$ (left) and $\varepsilon_{2}$ (right), respectively.

We study the dispersion curves of the plasmonic waves of the metal-dielectric-layered graphene structure using the method of recurrence relations developed in Ref.[29]. This approach offers an alternative of transfer matrix methods and besides that allows us to obtain analytical expressions of effective dielectric function of the structure.

We consider the Poisson's equation for the electrostatic potential as $\nabla^{2} \varphi(r, t)=0$ between graphene layers where $\varphi(\vec{r}, t)=\varphi(z) e^{i(q x-\omega t)}$ and the electrostatic potential between $I$ and $I+1$ layers as $\varphi_{l}(z)=A_{l} e^{-q(z-l d)}+B_{l} e^{q(z-l d)}$. Using the standard electrostatic boundary conditions at $z=l d$, we have

$$
\begin{aligned}
& A_{l-1} e^{-q d}+B_{l-1} e^{q d}=A_{l}+B_{l} \\
& -A_{l-1} e^{-q d}+B_{l-1} e^{q d}-\left(-A_{l}+B_{l}\right)=2 V_{q} \Pi(q, \omega)\left(A_{l-1} e^{-q d}+B_{l-1} e^{q d}\right)
\end{aligned}
$$

where $V_{q}=2 \pi e^{2} / \varepsilon_{0} q$ is the Fourier transform of the two-dimensional Coulomb interaction, $e$ the electron charge and $\Pi(q, \omega)$ is the graphene electron polarizability calculated in Refs.[30,31]. Equation (1) an be written as

$$
\left(\begin{array}{l}
A_{l} \\
B_{l}
\end{array}\right)=T\left(\begin{array}{l}
A_{l-1} \\
B_{l-1}
\end{array}\right)
$$




$$
T=\left(\begin{array}{cc}
\left(1+V_{q} \Pi(q, \omega)\right) e^{-q d} & V_{q} \Pi(q, \omega) e^{q d} \\
-V_{q} \Pi(q, \omega) e^{-q d} & \left(1-V_{q} \Pi(q, \omega)\right) e^{q d}
\end{array}\right)
$$

here, $T$ represents the transfer matrix connecting the coefficients at adjacent graphene layers with unit determinant and $\operatorname{Tr}(T)=2\left(\cosh q d-V_{q} \Pi(q, \omega) \sinh q d\right)=2 R$. From Eqs. $(2)$, it is possible to obtain the following linear recurrence relation of second order with constant coefficients,

$$
A_{l+2}-2 R A_{l+1}+A_{l}=0
$$

Recurrence relations given by Eq.(3) can be solved using standard methods, the solutions of which can be written in terms of the electrostatic amplitudes $A_{0}$ and $B_{0}$ as

and

$$
A_{l}=\frac{-1}{\sin \theta}\left[A_{0} \sin (l-1) \theta-A_{0} T_{11} \sin l \theta-B_{0} T_{12} \sin l \theta\right]
$$

$$
B_{l}=\frac{-1}{\sin \theta}\left[B_{0} \sin (l-1) \theta-B_{0} T_{22} \sin l \theta-A_{0} T_{21} \sin l \theta\right]
$$

where the coefficients $T_{n m}$ are the elements of the transfer matrix, the angle $\theta$ is defined as $\cos \theta=R,|R|<1$.

According to Fig. 1, the superlattice of $N$ graphene layers is terminated at the left by a metallic substrate separated by a dielectric film and, the electrostatic potential outside of the multilayer graphene structure is given as

$$
\varphi(z)=\left\{\begin{array}{c}
C e^{q z} \quad z<-\left(d_{m}+d_{s}\right) \\
N_{m} e^{-q z}+M_{m} e^{q z}-\left(d_{m}+d_{s}\right)<z<d_{s} \\
N_{s} e^{-q z}+M_{s} e^{q z}-d_{s}<z<0 \\
D e^{-q z} \quad z>(N-1) d
\end{array}\right.
$$


matching the electromagnetic boundary conditions at the interfaces $z=0, z=-d_{s}, z=-\left(d_{m}+d_{s}\right)$ and $z=(N-1) d$, leads the relationship between the electrostatic coefficients $A_{0}, B_{0}, A_{N-2}$ and $B_{N-2}$;

$\frac{A_{0}}{B_{0}}=\frac{\left(\varepsilon_{0}-\varepsilon_{s}+2 V_{q} \Pi(q, \omega)\right)+\left(\varepsilon_{s}+\varepsilon_{0}+2 V_{q} \Pi(q, \omega)\right) F(q, \omega) e^{-2 q d_{s}}}{\left(\varepsilon_{s}+\varepsilon_{0}-2 V_{q} \Pi(q, \omega)\right)-\left(\varepsilon_{s}-\varepsilon_{0}+2 V_{q} \Pi(q, \omega)\right) F(q, \omega) e^{-2 q d_{s}}}=G(q, \omega)$

$$
\begin{array}{r}
\frac{A_{N-2}}{B_{N-2}}=\frac{\varepsilon_{0}+\varepsilon_{2}-2 V_{q} \Pi(q, \omega)}{\varepsilon_{0}-\varepsilon_{2}+2 V_{q} \Pi(q, \omega)}=\frac{e^{2 q d}}{H(q, \omega)} \\
F(q, \omega)=\frac{\left(\varepsilon_{m}-\varepsilon_{1}\right)\left(\varepsilon_{s}+\varepsilon_{m}\right) e^{-2 q d_{m}}+\left(\varepsilon_{m}+\varepsilon_{1}\right)\left(\varepsilon_{s}-\varepsilon_{m}\right)}{\left(\varepsilon_{m}-\varepsilon_{1}\right)\left(\varepsilon_{s}-\varepsilon_{m}\right) e^{-2 q d_{m}+\left(\varepsilon_{m}+\varepsilon_{1}\right)\left(\varepsilon_{s}+\varepsilon_{m}\right)}}
\end{array}
$$

$$
H(q, \omega)=\frac{\varepsilon_{0}-\varepsilon_{2}+2 V_{q} \Pi(q, \omega)}{\varepsilon_{0}+\varepsilon_{2}-2 V_{q} \Pi(q, \omega)}
$$

with $\varepsilon_{m}(\omega)=\varepsilon_{\infty}-\omega_{p}^{2} / \omega^{2}$ the dielectric permittivity of the metal and $\omega_{p}$ and $\varepsilon_{\infty}$ the plasmon frequency and dielectric constant at infinite frequency, respectively. The condition for the collective oscillations of the coupled surface plasmon in metal via Coulomb interaction with the two-dimensional electron gas in graphene is the self sustaining oscillations in the electron density providing the electrostatic amplitudes $A_{m}$ and $B_{m} \neq 0$. Making use of Eqs.(6) and (7) in Eq.(4) the condition for no vanishing electrostatic potential through $A_{m}$ and $B_{m}$ is

$\varepsilon(q, \omega)=G(q, \omega) H(q, \omega)\left[\sin (N-3) \theta-T_{11} \sin (N-2) \theta\right]-[G(q, \omega)+$ $H(q, \omega)] T_{12} \sin (N-2) \theta-\left[\sin (N-3) \theta-T_{22} \sin (N-2) \theta\right] e^{2 q d}=0$ 
Equation (10) represents the generalized dielectric function of the stacked structure and is the condition to obtain the dispersion relation $\omega(q)$ of plasmon excitation in the metamaterial. As can be observed, the plasmon dispersion relation in general will depend on all the parameters of the metamaterials such as the number of graphene layers, the thickness and dielectric constants of both, the metal and dielectric films and the dielectric permittivity of the surrounding medium which play and important role for plasmonic sensing.

As a significant exemplification of the formalism introduced here we consider the strong Coulomb coupling between a monolayer graphene and the metal-dielectric structure. Under this approximation, the plasmon dispersion relation can be calculated from the zeros of the following dielectric function;

$$
\varepsilon(q, \omega)=\frac{\varepsilon_{s}-\varepsilon_{2}+2 V_{q} \Pi(q, \omega)}{\varepsilon_{s}+\varepsilon_{2}-2 V_{q} \Pi(q, \omega)} F(q, \omega)-e^{2 q d_{s}}
$$

Due to the phase-space constrains in graphene, there is a region of undamped plasmons satisfying the following condition $\gamma q<\omega<2 E_{f}-\gamma q$ and $\operatorname{Im} \Pi(q, \omega)=0$ thus, in order to obtain the plasmon modes, we proceed to use la long wavelength limit $(q->0)$ form of the graphene electron polarizability [31]

$$
\Pi(q, \omega)=\frac{E_{f}}{\pi} \frac{q^{2}}{\omega^{2}}\left(1-\frac{\omega^{2}}{4 E_{f}^{2}}\right)
$$

and a power expansion for the plasmon dispersion relation as in Refs. [32,33] i.e., $\omega(q)=c_{1} q+c_{2} q^{2}+\ldots$ The hybridization of charge oscillations in metal coupled with the graphene layer via many-body Coulomb interaction in the long wavelength limit lead and optical and acoustic plasmon modes; 


$$
\omega_{o}=\sqrt{c_{1}} q^{1 / 2}=\sqrt{\frac{4 e^{2} E_{f}+d_{m} \omega_{p}^{2}}{\varepsilon_{1}+\varepsilon_{2}}} q^{1 / 2}
$$

and

$$
\omega_{a c}=\sqrt{c_{2}} q=\sqrt{\frac{4 d_{s} d_{m} e^{2} E_{f} \omega_{p}^{2}}{\varepsilon_{s}\left(4 e^{2} E_{f}+d_{m} \omega_{p}^{2}\right)}} q
$$

The energy associated to the optical plasmon mode corresponds to the hybridization of the symmetric surface mode in metallic film and the optical graphene plasmon mode, note the this high energy plasmon branch depends substantially on the Fermi energy of the graphene, the thickness, the plasmon energy of the metallic film and the dielectric constants outside of the metal-dielectric graphene structure. These latter properties make this metamaterial a high sensitive $\mathrm{THz}$ surface plasmon resonance sensor. On the other hand, in the lower branch, the carriers in graphene oscillate out of phase with the metal surface plasmon generating surface acoustic plasmons exhibiting a linear dispersion with $q$ at long wavelength limit. Recently Lee et.al., have demonstrated a graphene acoustic plasmon resonator with nearly perfect absorption of incident infrared light [34]. Thus, this new metamaterial, metal-dielectric monolayer graphene could greatly improve ultrasensitive biosensors for optical diagnosis of diseases. It also follows from Eq.(14) that acoustic plasmon modes exist if the group velocity is greater than the Fermi velocity i.e., $c_{2}>\gamma^{2}$ and thus is not subject to Landau damping. This latter condition allow us to define a critical thickness of the dielectric film $d_{c}$ for which $c_{2}=\gamma^{2}$. By using this value of $c_{2} \ln$ Eq.(12) we obtain

$$
d_{c}=\frac{\varepsilon_{s} \gamma^{2}\left(4 e^{2} E_{f}+d_{m} \omega_{p}^{2}\right)}{4 d_{m} e^{2} E_{f} \omega_{p}^{2}}
$$


For thickness of the dielectric film larger of $d_{c}$ acoustic plasmon emerges above the electron-hole pair continuum for the metal-dielectric-graphene metamaterial.

Recently, Moradi [35] studied numerically the plasmonic characteristics of a monolayer graphene, which is coupled to a semi-infinite conductor separated by a dielectric layer, in this layered structure also two plasmon branches are presented, a higher energy frequency branch where the surface carriers oscillate in phase, optical mode and a lower branch with the electrons oscillating out of phase, acoustic plasmon mode.

In general, results for the plasmon dispersion can be presented by solving numerically Eq. (10) for a system consisting of a finite number of graphene layers $N$ coupling via Coulomb interaction with the electron gas in the metal as shown in Fig.1. However in the $\mathrm{THz}$ region, which is our interest, we take the long wavelength approximation. Under this approximation $\theta \sim q$ and Eq.(10) reduces to

$$
\begin{aligned}
& \varepsilon(q, \omega)=G(q, \omega) H(q, \omega)\left[\mathrm{N}-3-T_{11}(N-2)\right]-[G(q, \omega)+H(q, \omega)] T_{12}(\mathrm{~N}-2)- \\
& {\left[\mathrm{N}-3-T_{22}(\mathrm{~N}-2)\right] e^{2 q d}=0}
\end{aligned}
$$

whose solution for the plasmon optical mode is written as

$$
\omega_{o}^{2}=\frac{4 N e^{2} E_{f}}{\varepsilon_{1}+\varepsilon_{2}} q+\frac{d_{m} \omega_{p}^{2}}{\varepsilon_{1}+\varepsilon_{2}} q
$$

where the first term of the right hand in Eq.(15) represents the optical plasmon energy of a monolayer graphene times the graphene number of layers $N$, i.e., the electron density oscillations of the stacked graphene layers are in phase coupled via Coulomb interaction with the symmetric plasmon modes in the metal film. Equation (17) can also be obtained assuming an effective electron polarizability given as $N \Pi(q, \omega)$ In Eq.(11). This latter result is appropriate to study the optical properties in graphene-stacked layers, specifically; reflection, transmission and absorption of light in multilayer graphene. On the 
other hand, the acoustic plasmon branch due to the asymmetric behavior, it must to be calculated numerically for the metal-dielectric-multilayer graphene structure.

\section{Numerical results}

The system corresponding to metal-graphene plasmonic metamaterial where graphene and metal are separated by a dielectric spacer is shown in Fig.(1). When the graphene layers are far apart Coulomb interaction between them can be neglected and collective electron excitations consist in plasmons excited in metal-dielectric-monolayer graphene given by Eqs.(13-14). Nevertheless, when Coulomb interaction between graphene layers are turn on new optical and acoustic plasmon branches emerge in the regime which we are interested i.e., $\omega>\gamma q$ and $E_{f}>\omega$. The dependence of acoustic plasmons as function of the wave vector is shown in Fig.(2) for different number of graphene layers. As can been observed, provided that the thickness of the dielectric film satisfies Eq.(15), the acoustic plasmon energy is free Landau damping in the long wave limit and it increase with the number of graphene layers on the metal-insulator stack. However, as $q$ increase, it is expected that the plasmon dispersion relation decay into the continuum of the interband single particle excitation region.

Surface plasmons in graphene can be significantly influenced by many-particle involving interaction between electrons and plasmons and besides that, they can be tuned continuously by manipulating the thickness of the metal, the dielectric properties of the external medium, etc. The dispersion curves of the surface plasmons on graphene metaldielectric structures reveal these essential features. Therefore, the interaction of light with surface plasmons can be enhanced leading a great ability for biological sensing applications. Usually surface plasmon can be excited via evanescent waves in the Kretschmann configuration, utilizing high-index prisms, where the wavevector matching between incident light and surface plasmon is compensated. Once surface plasmon is excited in the Kretschmann configuration, a sharp minimum is observed in the reflection coefficient versus incident angle (or wavelength) curve. 
In the present work the p-polarized incident light is on the metal substrate, and in order to determine the reflectance of plasmon resonances as a function of the incident angle, we solve the Fresnel problem for the metal-dielectric-graphene structure as in Ref.[36]. If the $x-y$ plane is the interface plane, for wave propagation in the $x$ direction only, for $p$ polarization the magnetic field is polarized along the $y$ direction and can be written in the form $\boldsymbol{H}=\left(0, U e^{-i k z}+V e^{i k z}, 0\right) \boldsymbol{e}^{i \kappa x-i \omega t}=(0, \Phi, 0)$ and the electric field associated with this electromagnetic wave is $\boldsymbol{E}=-c / i \omega \varepsilon\left(-\partial_{z} \Phi, 0, \partial_{x} \Phi\right)$ with $k^{2}=\varepsilon \omega^{2} / c^{2}-\kappa^{2}$ and $c$ the incident light velocity. Furthermore, the propagation of light across the set of interfaces formed by a graphene layers and the metal and dielectric slabs, must satisfy the standard electromagnetic boundary conditions i.e., the tangential component of $\boldsymbol{E}$ is continuous and $\boldsymbol{H}$ discontinuous. Thus, the relationship between the field components $U$, $V$ at the first and last interface are related as:

$$
\left(\begin{array}{l}
U_{1} \\
V_{1}
\end{array}\right)=M\left(\begin{array}{l}
U_{N+1} \\
V_{N+1}
\end{array}\right)
$$

where $M$ is the characteristic transfer matrix of the combined metal-dielectric- stack graphene structure and can be obtained from the following relation for the p-polarization light [37]

$$
M=R_{1 \rightarrow 2} P\left(d_{1,2}\right) R_{2 \rightarrow 3} P\left(d_{2,3}\right) \ldots P\left(d_{N-1 N}\right) R_{N \rightarrow N+1}
$$

where the electric and magnetic fields in different layers are related through the propagation matrix $P\left(d_{m, m+1}\right)$

$$
P\left(d_{m, m+1}\right)=\left(\begin{array}{cc}
e^{-i k_{m+1} d_{m, m+1}} & 0 \\
0 & e^{i k_{m+1} d_{m, m+1}}
\end{array}\right)
$$

and the transmission matrix 


$$
R_{m \rightarrow m+1}=\frac{k_{m+1}}{2 \varepsilon_{m+1}}\left(\begin{array}{cc}
\frac{\varepsilon_{m}}{k_{m}}+\frac{\varepsilon_{m+1}}{k_{m+1}}+\frac{4 \pi}{\omega} \sigma(\omega) & -\frac{\varepsilon_{m}}{k_{m}}+\frac{\varepsilon_{m+1}}{k_{m+1}}-\frac{4 \pi}{\omega} \sigma(\omega) \\
-\frac{\varepsilon_{m}}{k_{m}}+\frac{\varepsilon_{m+1}}{k_{m+1}}-\frac{4 \pi}{\omega} \sigma(\omega) & \frac{\varepsilon_{m}}{k_{m}}+\frac{\varepsilon_{m+1}}{k_{m+1}}-\frac{4 \pi}{\omega} \sigma(\omega)
\end{array}\right)
$$

Here $\sigma(\omega)$ is the surface conductivity of graphene which takes into account the contribution of the interband and intraband electronic transitions as

$$
\sigma_{\text {intra }}=\frac{2 i e^{2} k_{B} T}{\pi \hbar^{2}(\omega+i \Gamma)} \ln \left[2 \cosh \left(\frac{E_{f}}{2 k_{B} T_{0}}\right)\right]
$$

and

$$
\sigma_{\text {inter }}=\frac{e^{2}}{4 \hbar}\left[\frac{1}{2}+\frac{1}{\pi}+\arctan \left(\frac{\hbar \omega-E_{f}}{2 k_{B} T_{0}}\right)-\frac{i}{2 \pi} \ln \frac{\left(\hbar \omega+E_{f}\right)^{2}}{\left(\hbar \omega-E_{f}\right)^{2}+\left(2 k_{B} T_{0}\right)^{2}}\right]
$$

where $k_{B}$ is the Boltzmann's constant, $\hbar$ is the reduced Planck's constant, $T_{O}$ is the temperature, $\omega$ is the angular frequency of incident light and $\Gamma$ is the relaxation frequency. Notice that in the metallic film and the dielectric spacer in the metamaterial $\sigma(\omega)=0$. Once the transfer matrix is known, we can calculate the optical properties of the multi-layer graphene on metal dielectric substrates. Assuming the incident light is on the metallic slab it can be easily shown that the reflection coefficient is given by the elements of the matrix $M, r=M_{21} / M_{11}$ and the reflectance $R=|r|^{2}$.

In Fig.(3) the reflectance of the metal-dielectric-graphene metamaterial is depicted as a function of the angle of the incident radiation for dielectric thickness increasing from 1-10 $\mathrm{nm}$. The results exhibit a shift of the sharp minimum reflectivity indicating a surface plasmon excitation in the Kretschmann configuration without variation of its amplitude. 
For three different dielectric thicknesses, the plasmonic angles shift from $43^{\circ}$ to $44.7^{\circ}$, therefore as can be noted the hybrid metamaterial used as biosensor is very sensitive to changes of the dielectric film thickness with optimum metallic thickness of $60 \mathrm{~nm}$ and incident electromagnetic radiation of $\lambda=632 \mathrm{~nm}$ with a dielectric constant of the prism $\varepsilon_{1}=2.25$ and the sensing medium $\varepsilon_{2}=1$. Figure (4) shows the variations of the reflectivity with the number of the graphene layers $N$ and the thickness of the dielectric film $d_{s}=5 \mathrm{~nm}$. These results indicate that the number of graphene layers can be optimized to enhance the performance and sensibility of the hybrid metamaterial structures-based surface plasmon biosensor. As can be seen, when the number of graphene layers increases there is a shift of the resonance peak and a reduction in the amplitude of the resonance dip. Thus, a complete transfer of energy of the incident radiation corresponds to one graphene layer.

Surface plasmon resonance can be also used to detect biological molecules. This feature is used to design optical biosensors that can measure the refractive index when the biomolecules become adsorbed on the graphene surface and create a layer of refractive index higher than that of the air ( $\varepsilon_{2}$ in Fig.(1)) resulting in a change in the resonance angle. Fig.(5) plots the theoretical reflectivity against the resonance angle for different refractive index of the sensing medium. As can be seen there is an increase in the width of the reflectance curve and a shift in the resonance angle of the minimum reflectivity with increasing refractive index of the sensing medium. Therefore, this plasmonic metamaterials are very sensitive to the changes of refractive index of the dielectric media in the vicinity of the graphene layer.

\section{Conclusions}

In this study, the collective electronic excitations in metal-dielectric-layered graphene structure were investigated. Long-range Coulomb interactions in the metamaterial lead new set spectra of surface plasmons, which depend on a certain characteristic parameters of each material in metal-dielectric-layered graphene structure. At long wavelength $(q \rightarrow 0)$ 
the acoustic plasmons emerge in this metamaterial structure characterized by a linear dependence on the momentum, which can be observed by electron energy los spectroscopy (EELS). Recently [34], new acoustic plasmon resonators have been proposed to excite and manipulate the interaction between field confinement associated with these plasmonic modes and free-space light. Also, this metamaterial proposed in this work supports high-energy optical plasmons, which can be excited by light using the Kretschmann coupling configuration. Since a high performance of surface plasmon resonance biosensor should exhibit large depth of dip (small reflectivity), the minimum of the reflectivity in the hybrid configuration can be manipulated dynamically by changing, the thickness of the dielectric film, the dielectric properties of the environment, the number of graphene layers etc. which is an a efficient method to realize high sensing properties for the metamaterial. As an application, we have shown that metal-dielectricgraphene stack is a sensitive $\mathrm{THz}$ plasmonic biosensor to study graphene layer interactions with biomolecules characterized with their own dielectric permittivity.

Funding: This work is partially supported by Consejo Nacional de Ciencia y Tecnologia (Conacyt), Mexico under grant 254414.

Author Contributions: Each author contributed equally.

Conflict of Interest: The authors declare no conflict of interest.

Availability of Data: No Applicable

Code Availability: No Applicable 


\section{References}

[1]. S. Hayashi and T. Okamoto, Plasmonics: visit the past to know the future, J. Phys. D: Appl. Phys. 45 (2012) 433001

[2] J. Shang, L. Zhang and W Xu, Surface plasmon polariton: Physics and applications, , J. Phys. D: Appl. Phys. 45 (2012) 113001

[3] S.S. Verma, Plasmonics in Nanomedicine: A Review, Global J. Nanomedicine, 4 (2018) 1

[4] M.I. Stockman, K. Kneipp, S. I. Bozhevolnyi, S. Saha, A. Dutta, J. Ndukaife, N. Kinsey, H. Reddy, U. Guler, V. M. Shalaev, Roadmap on plasmonics, J. Optics 20 (2018) 043001

[5] X. Han, K. Liu and C. Sun, Plasmonics for Biosensing, Materials 12 (2019) 1411

[6] S. K. Sekatskii 1, A. Smirnov, G. Dietler, M. N. E. Alam, M. Vasiliev and K. Alameh, Photonic Crystal-Supported Long-Range Surface Plasmon-Polaritons Propagating Along High-Quality Silver Nanofilms , Appl. Sci. 8 (2018) 248

[7] V. G. Kravets, R. Jalil, Y.-J. Kim, D. Ansell, D. E. Aznakayeva, B. Thackray, L. Britnell, B. D. Belle, F. Withers, I. P. Radko, Z. Han, S. I. Bozhevolnyi, K. S. Novoselov, A. K. Geim, and A. N. Grigorenko, Graphene-protected copper and silver plasmonics, Sci. Rep. DOI:

\section{$10.1038 /$ srep05517}

[8] Z. Li, K. Yao, F. Xia, S. Shen, J. Tian and Y. Liu, Graphene Plasmonic Metasurfaces to Steer Infrared Light, Sci. Rep. DOi: 10.1038/srep12423

[9] C. Liu, Y. Bai, J. Zhou, Q. Zhao and L. Qiao, A Review of Graphene Plasmons and its Combination with Metasurface, J. Korean Ceramic Soc. 54 (2017) 342.

[10] E. Galiffi, J.B. Pendry and P.A. Hudabro, Broadband Tunable THz Absorption with Singular Graphene Metasurfaces, ACS Nano 12 (2018) 1006

[11] E. Pisano, C.E. Garcia-Ortiz, F. Armenta-Monzon, M. Garcia-Mendez and V. Collo, Efficient and Directional Excitation of Surface Plasmon Polaritons by Oblique Incidence on 
Metallic Ridges, Plasmonics 13 (2018) 1935

[12] C. Shi, X. He, J. Peng, G. Xiao, F. Liu, F. Lin, H. Zhang, Tunable terahertz hybrid graphene-metal patterns metamaterials, Optics and Laser Technology, 144 (2019) 28

[13] S. Huang, C. Song, G.Zhang, and H. Yuan, Graphene plasmonics: physics and potential applications, Nanophotonics 6 (2017) 1191

[14] M.S. Zare, N. Nozhat and R. Rashiditabar, A strong controllable absorber using graphene- metal nanostructures, J. Mod. Opt. 66 (2019) 7

[15] L. Peng, X.F. Li, X. Gao, X. Jiang and S.M. Li, Methodology for the design of a multifunctional device with switchable absorption and polarization conversion modes by graphene and metallic metasurfaces, Op. Matt. Ex. 9 (2019) 687

[16] H.J. Zhang, G.G. Zheng, Y.Y. Chen, and L.H. Xu, Broadband and wide angle near-unity absorption in graphene-insulator-metal thin film stacks, Superlattices and Microstructures, 117 (2018) 137

[17] S. Benaziez, Z. Dibi and N. Benaziez, Reflectivity Optimization of the SPR Graphene Sensor, Nanopages, DOI: 10.1556/566.2018.0023

[18] Y. Huang, S. Zhong, Y. Shen, L. Yao, Y Yu and Daxiang Cui, Graphene/Insulator Stack Based Ultrasensitive Terahertz Sensor With Surface Plasmon Resonance, IEEE Photonics Journal 9 (2017) DOI:10.1109/JPHOT.2017.2765182

[19] P. Suvarnaphaet and S. Pechprasarn, Graphene-Based Materials for Biosensors: A Review , 17(2017) 2161

[20] X. Chen, W. Fan, C. Song, Multiple plasmonic resonance excitations on graphene metamaterials for ultrasensitive terahertz sensing, Carbon 133 (2018) 416

[21] Y. Huang, S. Zhong, Y.C. Shen, Y. Yub and D. Cui, Terahertz phase jumps for ultrasensitive graphene plasmon sensing, Nanoscale, 10 (2018) 22466

[22] H. Vahed and C. Nadri, Ultra-sensitive surface plasmon resonance biosensor based on MoS2-graphene hybrid nanostructure with silver metal layer, Optical and Quantum Electronics 51 (2019) 20

[23] S. Chen, C. Lin, Figure of merit analysis of graphene based surface plasmon resonance biosensor for visible and near infrared, Optics Communications 435 (2019) 102

[24] S.Niknam, M. Yazdi andS. B. Amlashi, enhanced Ultra-sensitive Metamaterial 
Resonance sensor based on Double Corrugated Metal stripe for terahertz sensing Sci. Rep. 9 (2019) 751

[25] L. Han, X. He, L. Ge, T. Huang, H. Ding and C. Wu, Comprehensive Study of SPR Biosensor Performance Based on Metal-ITO-Graphene/TMDC Hybrid Multilayer, Plasmonics (2019) , doi.org/10.1007/s11468-019-01004-w

[26] N.J.M. Horing, Coupling of graphene and surface plasmons, Phys. Rev. B 80 (2009) 193401

[27] G. Gonzalez de la Cruz, Role of metallic substrate on the plasmon modes in doublelayer graphene structures, Solid State Commun. 213 (2015) 6

[28] G. Gumbs, A. Lurov, J.Y. Wu, M.F. Lin and P. Fekete, Plasmon Excitations of Multi-layer Graphene on a Conducting Substrate, Sci. Rep.6 (2016), 21063

[29] G. Gonzalez de la Cruz, Bulk and surface plasmons in graphene finite superlattices, Superlattices and Microstructures, 125 (2019) 315

[30] B. Wunsch, T. Stauber, F. Sols, F. Guinea, Dynamical Polarization of graphene at finite doping, New. J. Phys. 8, (2006), 318

[31] E.H. Wang, S. DasSarma, Dielectric function, screening, and plasmons in twodimensional graphene, Phys. Rev. B 75, (2007), 205418

[32] G.E. Santoro and G. Giuliani, Acoustic plasmons in a conducting double layer, Phys. Rev. B 37 (1988) 937

[33] R. E. V. Profumo, R. Asgari, M. Polini and A. H. MacDonald, Double-layer graphene and topological insulator thin-film plasmons, Phys. Rev. B 85 (2012) 085443

[34] I.H. Lee, D. Yoo, P. Avouris, T. Low, and S.H. Oh, Graphene acoustic plasmon resonator for ultrasensitive infrared spectroscopy, Nat. Nanotech. doi.org/10.1038/s41565-019-036-8

[35] A. Moradi, Plasmonic waves of graphene on a conducting substrate , J. Mod. Opt. 66 (2019) 353

[36] R.A. Define Graphene Optics: Electromagnetic Solution of Canonical Problems (2016) IOP Publishing, Bristol BS1 6HG, UK

[37] Tianrong Zhan, Xi Shi, Yunyun Dai, Xiaohan Liu and Jian Zi, Transfer matrix method for optics in graphene layers, J. Phys.: Condens. Matter 25 (2013) 215301 


\section{Figure captions}

Fig. 1: Schematic representation of graphene stacks separated a distance $d$ embedded in a medium with dielectric permittivity $\varepsilon_{0}$ on a metal-dielectric substrate with metal thickness $d_{m}$ and dielectric thickness $d_{s}$ surrounded by a dielectric constant $\varepsilon_{1}$ and $\varepsilon_{2}, \varepsilon_{m}$ represents the dielectric permittivity of the metallic film with $\omega_{p}=1.3710^{16} / \mathrm{sec}$. and $E_{f}=0.15 \mathrm{eV}$

Fig.2: Dispersion relation of the acoustic plasmon for different number of graphene layers and dielectric thickness $d_{s}=10 \mathrm{~nm}, d_{m}=60 \mathrm{~nm}, \varepsilon_{1}=2.25, \varepsilon_{2}=1, \lambda=632 \mathrm{~nm}$. The others parameters used are the same as in Fig.1

Fig.3: Reflectivity spectra of the metal-dielectric-graphene metamaterial as a function of the incident angle for different thickness of the dielectric film. The other parameters are the same as Fig.2

Fig.4: Reflectivity spectra of the metal-dielectric-graphene metamaterial as a function of the incident angle for different number of the graphene layers. The other parameters are the same as Fig. 2.

Fig.5: Reflectivity spectra curves as a function of the incident angle for different dielectric permittivity of adsorbed biomolecules on the monolayer graphene surface. Same parameters as Fig. 2 


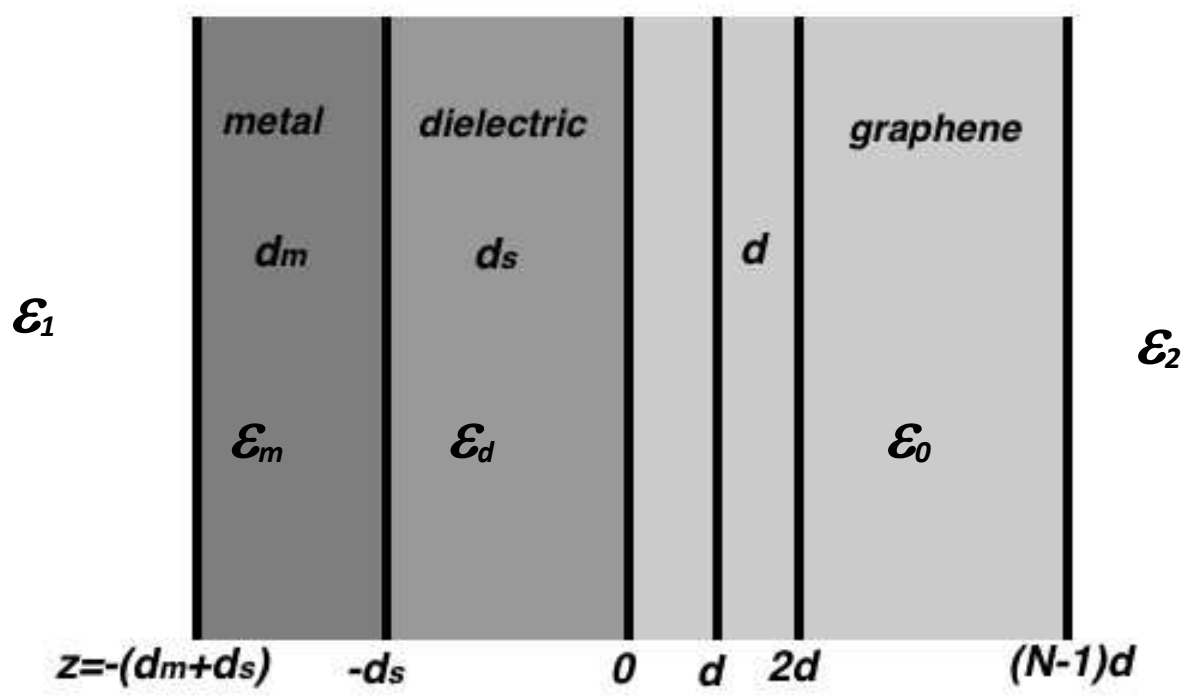

Fig. 1 


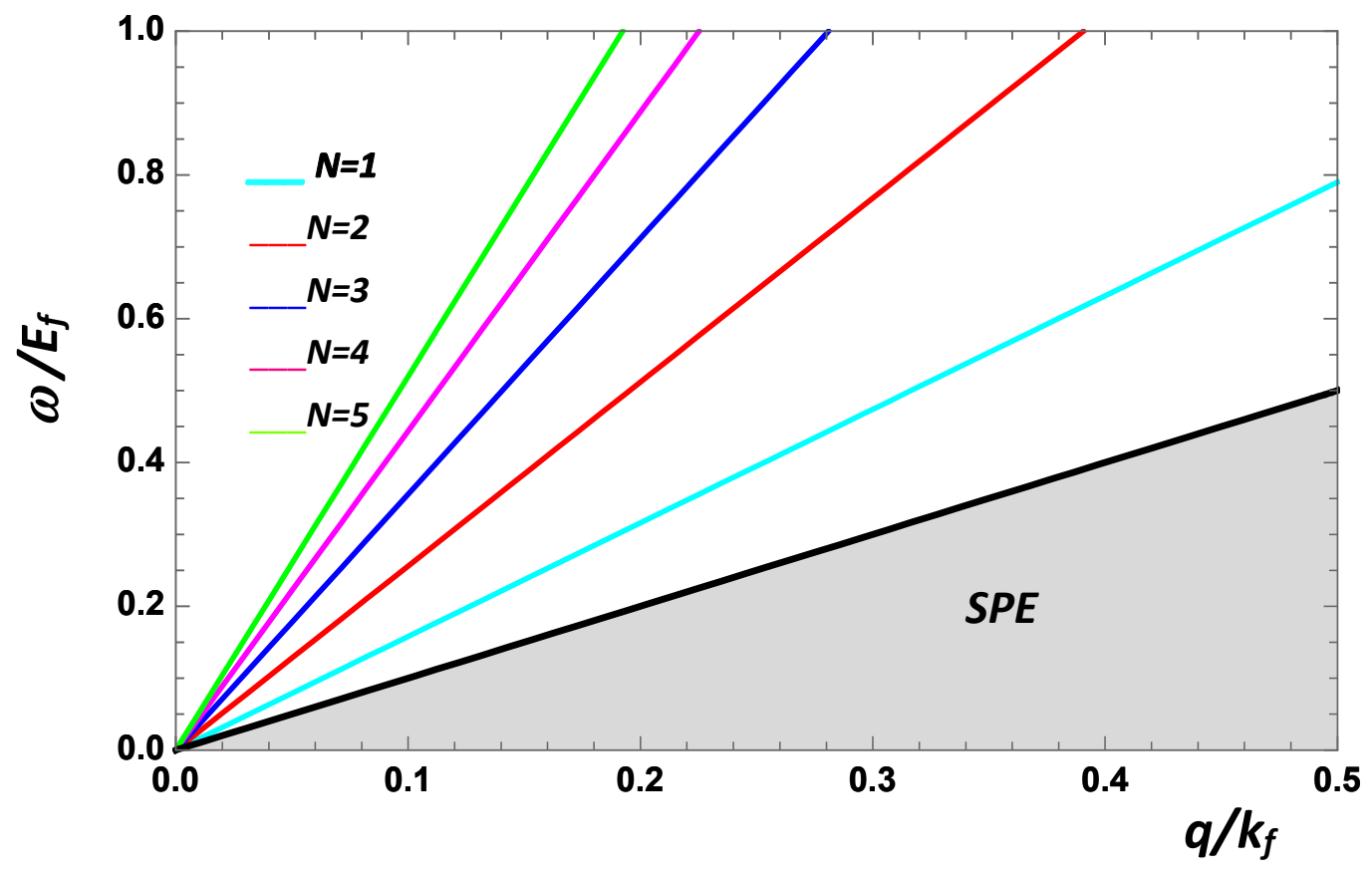

Fig. 2 


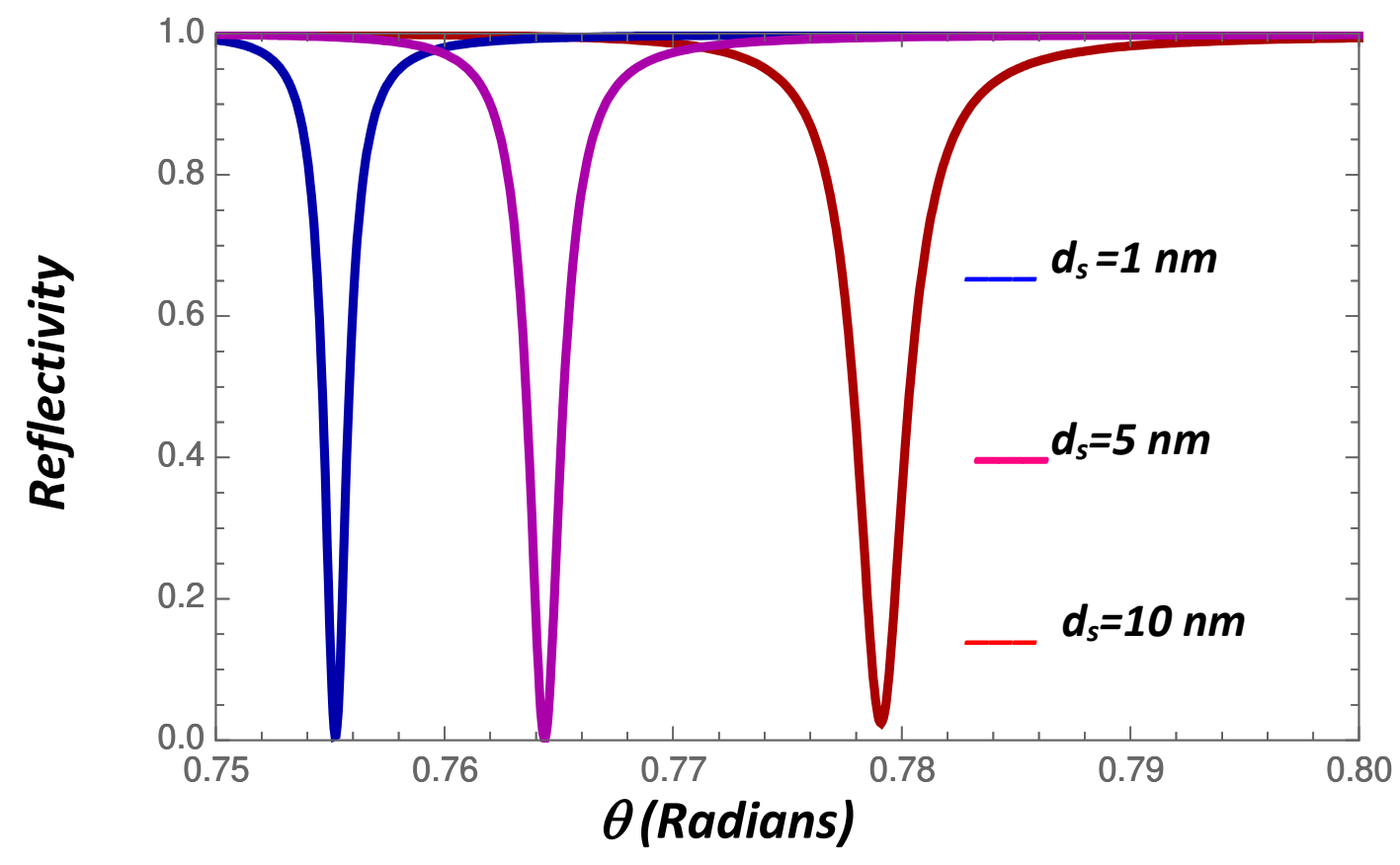

Fig.3 


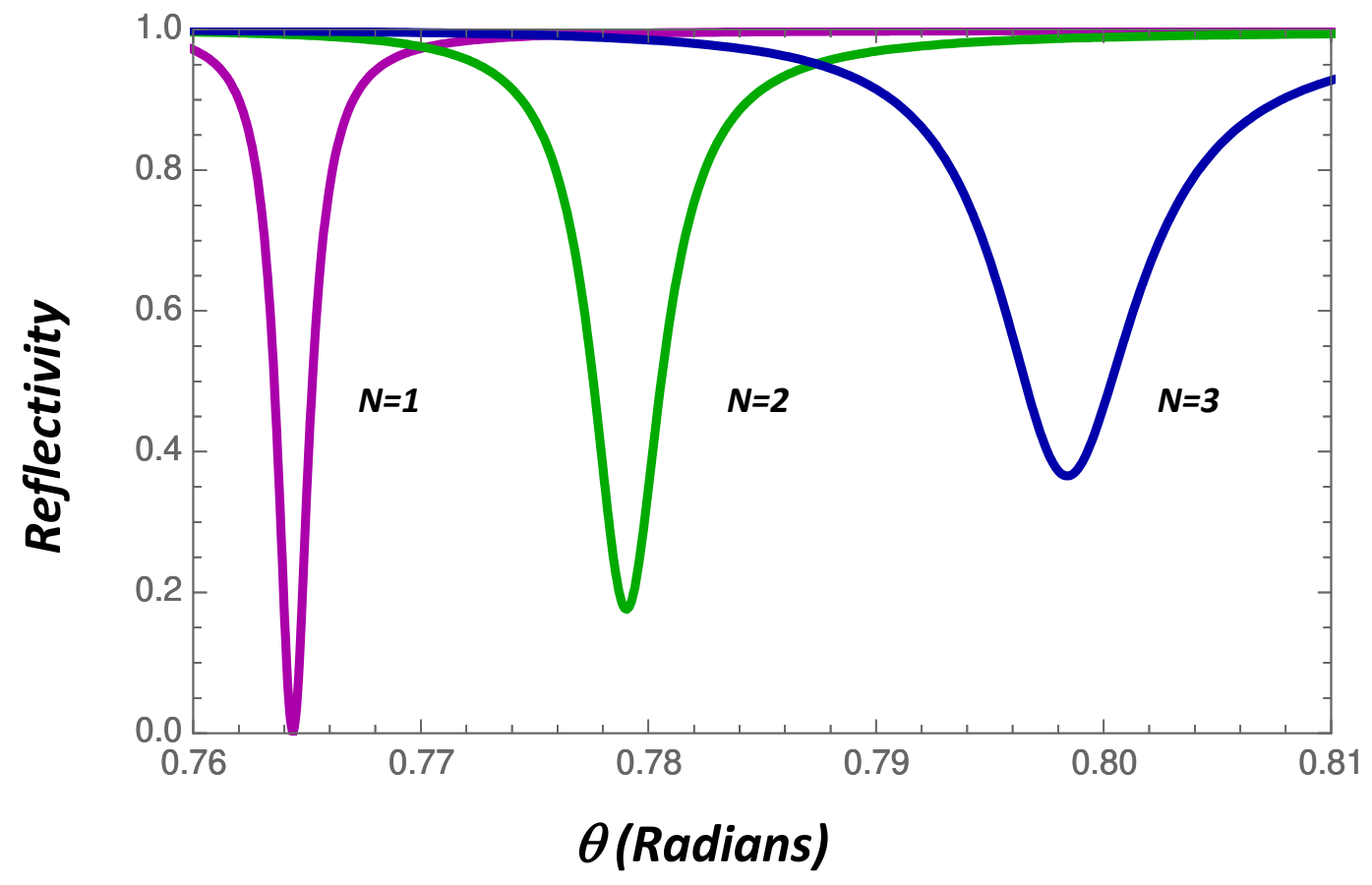

Fig.4 


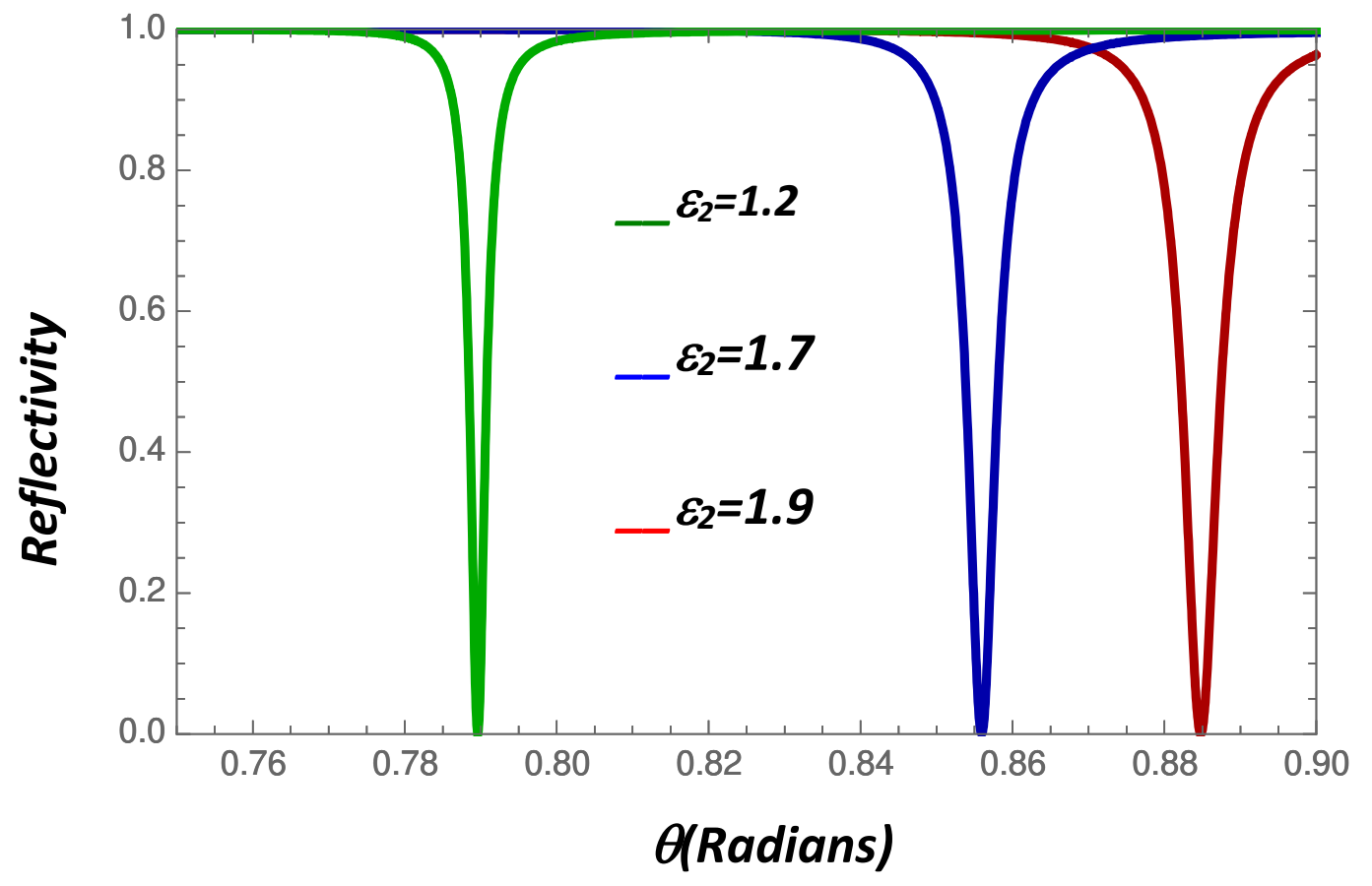

Fig.5 
Figures

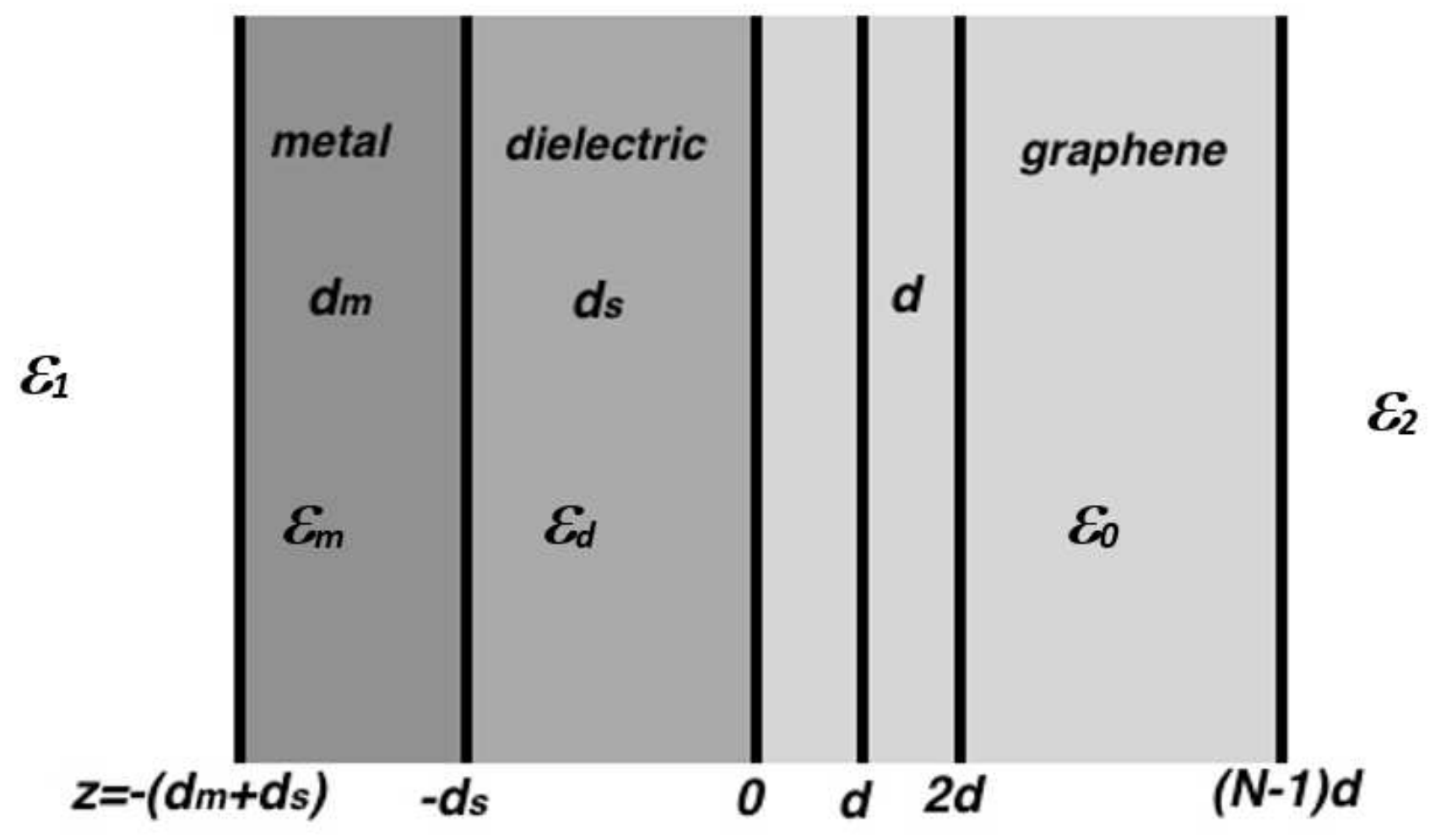

Figure 1

See manuscript for full figure caption. 


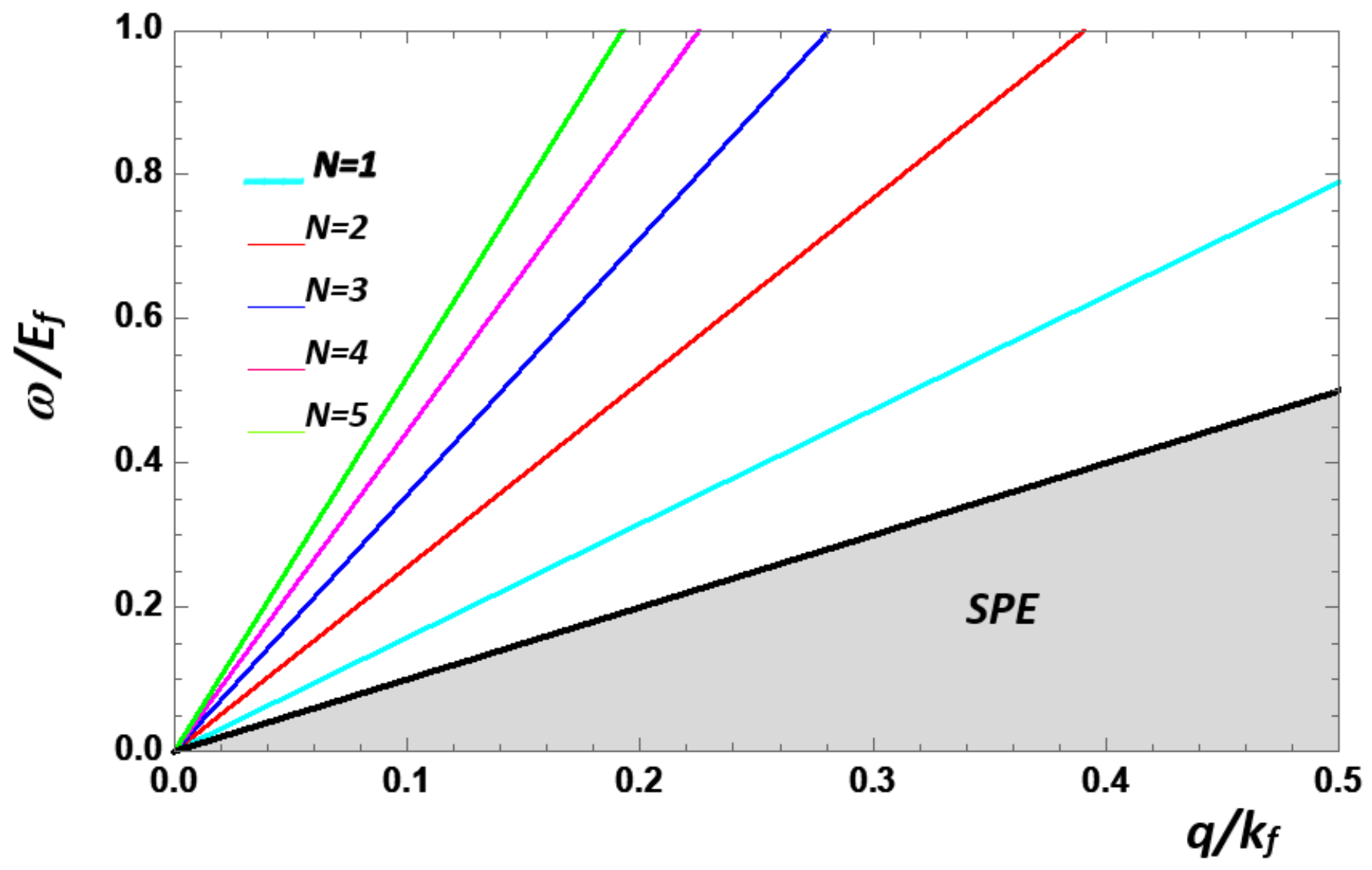

Figure 2

See manuscript for full figure caption. 


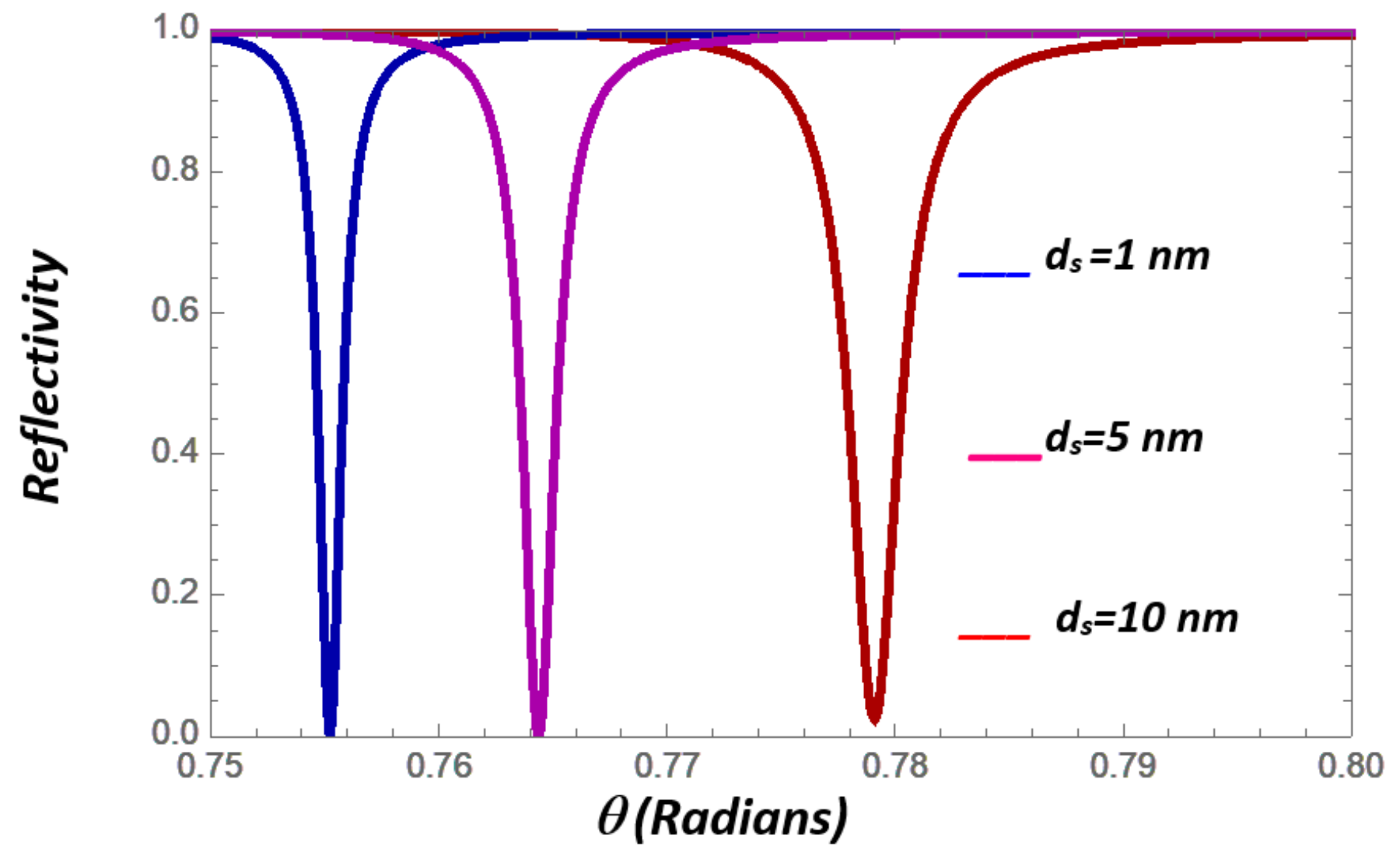

Figure 3

See manuscript for full figure caption. 


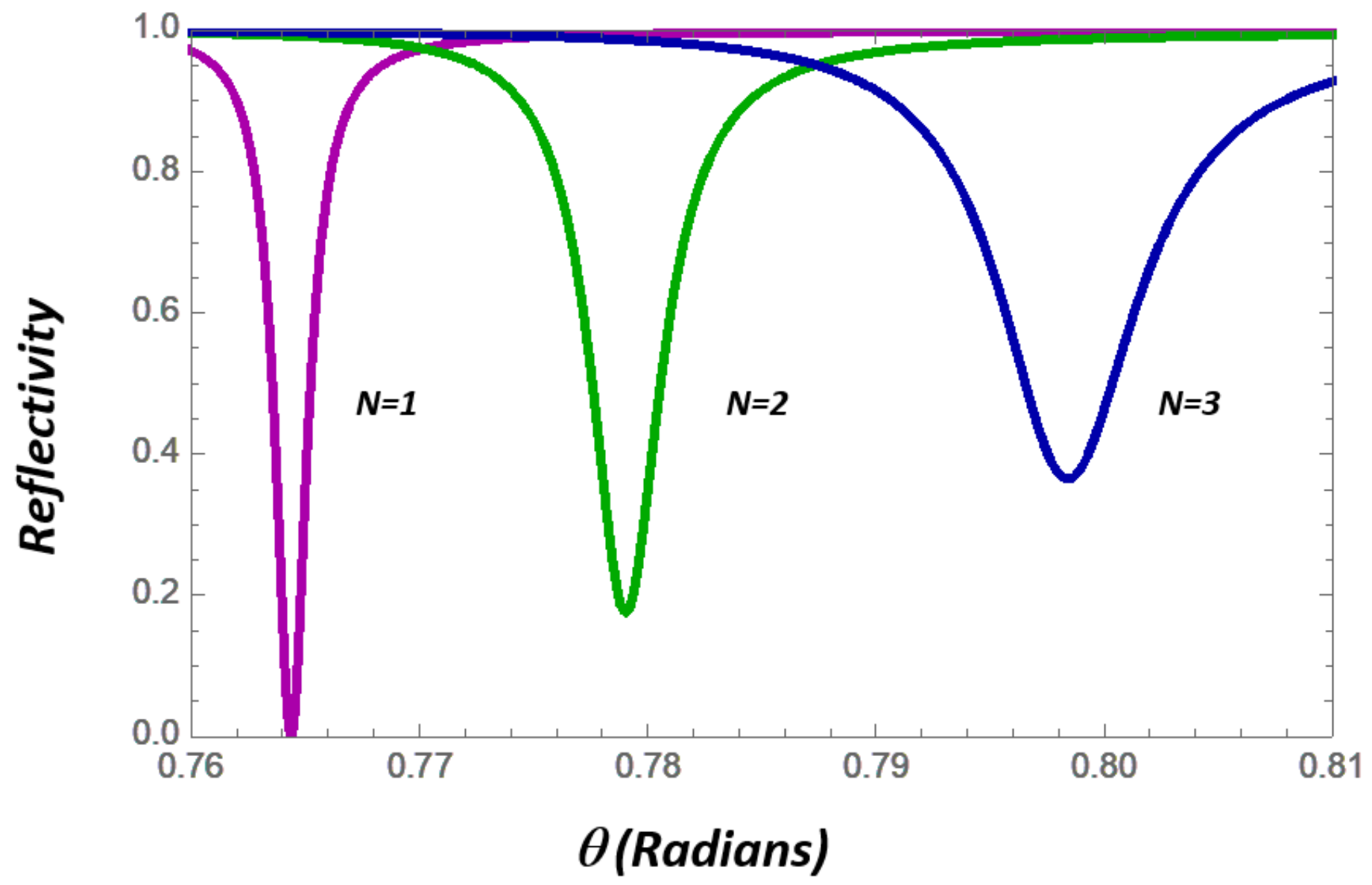

Figure 4

See manuscript for full figure caption. 


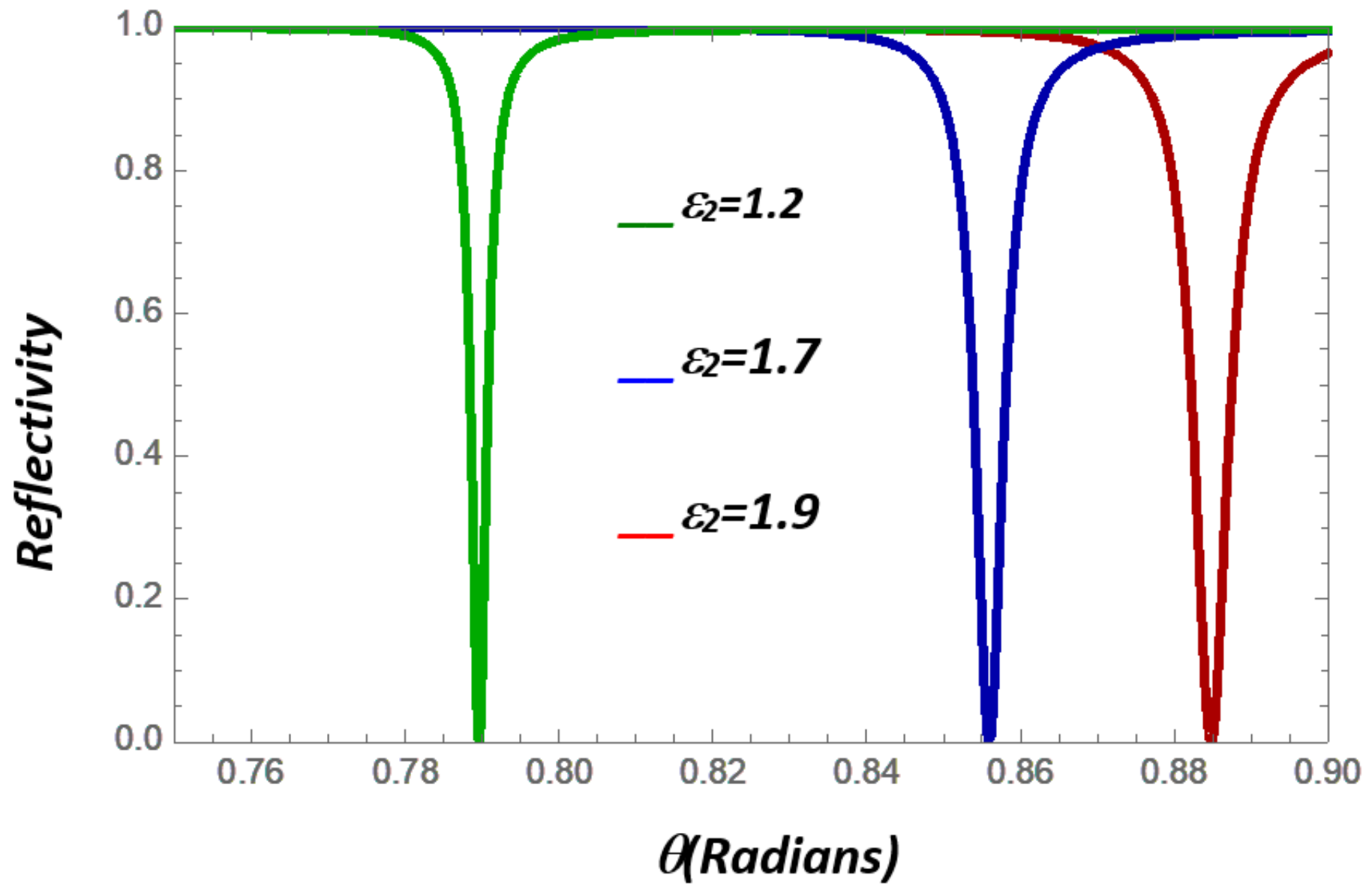

Figure 5

See manuscript for full figure caption. 